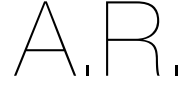

ARTIGO DE REVISÃO

${ }^{1}$ Escola Superior de

Tecnologia da Saúde do

Instituto Politécnico de

Coimbra,

Rua 5 de Outubro - SM

Bispo,

Apartado 7006

3046-854 Coimbra,

Portugal

"Endereço para correspondência:

Carolina Gomes

Rua do Forno, n. ${ }^{5} 53$ - Pardielas,

2240-371 Ferreira do Zêzere,

Portugal

carolinaramalhogomes@

hotmail.com

Histórico do artigo:

Recebido a 14 de maio de 2020 Aceite 25 de setembro de 2020

\title{
O FITATO E A BIODISPONIBILIDADE DE FERRO NAS LEGUMINOSAS
}

\author{
PHYTATE AND IRON BIOAVAILABILITY IN LEGUMES
}

Bruna Pinheiro'; Carolina Gomes; ${ }^{1}$ Ana Lúcia Baltazar ${ }^{1}$

RESUMO

As leguminosas são ricas em proteínas, hidratos de carbono, fitoquímicos, vitaminas, minerais e anti-nutrientes. Os anti-nutrientes reduzem a biodisponibilidade de vitaminas e minerais. O fitato é um inibidor dominante da biodisponibilidade de minerais, como o ferro (Fe). O Fe tem um papel crucial em vários processos metabólicos, como a síntese de hemoglobina, hormonas, DNA, tecido conjuntivo e muscular, tendo um papel crucial na produção de energia e transporte de oxigénio no sangue. Assim, a presença de fitato pode causar deficiência de Fe e várias consequências para o organismo. Portanto, a redução ou eliminação deste anti-nutriente é essencial para melhorar a utilização biológica de leguminosas e reduzir possíveis efeitos adversos.

O objetivo desta revisão é entender o metabolismo, efeitos e doses do fitato. Além disso, outro objetivo é mencionar estratégias que reduzam os efeitos do fitato, melhorem a biodisponibilidade do Fe e previnam a deficiência deste micronutriente.

O fitato, o complexo de ácido fítico e elementos minerais, é um agente quelante que reduz a biodisponibilidade mineral. Este tem um efeito inibitório no Fe quando a razão molar fitato/Fe é maior do que 1. As consequências da deficiência de Fe incluem diminuição do desempenho físico e cognitivo, depressão e fadiga. A demolha, a germinação, a fermentação e o tratamento térmico reduzem o conteúdo de fitato, aumentando assim a biodisponibilidade do Fe. A biofortificação melhora o status de Fe e parece melhorar as consequências da deficiência de Fe, tais como a capacidade física e função cognitiva.

Pesquisas futuras são necessárias para estudar outras variedades de leguminosas e em combinação com vários alimentos biofortificados, como cereais. Além disso, são necessários mais estudos para avaliar o desempenho físico e cognitivo, para desenvolver a biofortificação e melhorar a saúde das populações.

\section{PALAVRAS-CHAVE}

Absorção de ferro, Anti-nutrientes, Biodisponibilidade de ferro, Fitato, Leguminosas

\section{ABSTRACT}

Legumes are rich in proteins, carbohydrates, phytochemicals, vitamins, minerals and antinutrients. The antinutrients compounds reduce the bioavailability of micronutrients, like phytate. Iron (Fe) has a crucial role in several metabolic processes, such as hemoglobin' synthesis, hormones, DNA, conjunctive and muscle tissue, with a critical role in the production of energy and oxygen' transportation. The presence of phytate could cause Fe deficiency and several consequences for the organism. Therefore, the reduction or elimination of this antinutrient, it's essential to improve the biological utilization of legumes and to reduce possible adverse effects. This review aims to understand the phytate metabolization, effects and doses, and to identify strategies that can reduce the effects of phytate, to improve Fe bioavailability.

Phytate, the complex of phytic acid and mineral elements, is a chelating agent that reduces mineral bioavailability. It has an inhibitory effect on Fe when the molar ratio phytate/Fe is higher than 1. The consequences of Fe deficiency include decreased physical and cognitive performance, depression and fatigue. Soaking, germination, fermentation and heat treatment reduce phytate content, therefore increasing Fe bioavailability. Biofortification improves Fe status and seems to enhance the consequences of Fe deficiency, such as physical ability and cognitive function.

Future research is necessary to study different varieties of legumes and in combination with various biofortified foods, like cereals. Besides that, more studies are needed to assess physical and cognitive performance, to develop biofortification and improve the health of populations.

KEYWORDS

Iron absorption, Anti-nutrients, Iron bioavailability, Phytate, Legumes 


\section{INTRODUÇÃO}

As leguminosas têm benefícios para a saúde humana, pois fornecem fitoquímicos e outros constituintes químicos com atividades antioxidantes, que reduzem o risco de doenças como obesidade, hipertensão, diabetes e cancro e melhoram a função fisiológica, como por exemplo a saúde intestinal $(1,2)$.

As leguminosas são ricas em proteínas e aminoácidos essenciais (especialmente a lisina), hidratos de carbono, fitoquímicos (com propriedades antioxidantes, analgésicas e neuroprotetoras), vitaminas e minerais, como o folato, niacina, tiamina, cálcio, ferro $(\mathrm{Fe})$ e zinco (3-8). A concentração de nutrientes varia consideravelmente entre as leguminosas, variedades e locais dos grãos (8). Contêm também antinutrientes como o fitato, polifenóis como os taninos, oxalato e inibidores de protease, que reduzem a biodisponibilidade de vitaminas e minerais, inibindo a sua absorção (3-7, 9). Portanto, a redução ou eliminação destes anti-nutrientes é importante para melhorar a utilização biológica das leguminosas e para reduzir ou até eliminar os efeitos destes (3).

A biodisponibilidade é a proporção do conteúdo total de nutrientes de um alimento disponível, para funções metabólicas normais e indica a quantidade de um nutriente que é absorvida pelo corpo, depois da digestão gastrointestinal (6, 9). Muitos minerais não são eficientemente absorvidos na dieta, por exemplo, o Fe (<1 a 30\%). Este fenómeno deve ser tido em conta na preparação de alimentos para reduzir deficiências de micronutrientes, como o Fe, que leva à anemia (9).

A deficiência de Fe é uma das deficiências de micronutrientes mais comuns no mundo, afetando mais de $30 \%$ da população mundial, principalmente crianças e mulheres. De acordo com os dados de mortalidade da Organização Mundial da Saúde (OMS), a anemia por deficiência de Fe causa 17.000 mortes por ano em mulheres em idade reprodutiva, com mais de $70 \%$ dessas mortes ocorrendo em África $(4,8,10-13)$.

O Fe é necessário em vários processos metabólicos como síntese de hemoglobina, hormonas e tecido conjuntivo e está presente no tecido muscular como mioglobina $(4,14)$. Assim, o Fe tem um papel vital como transportador de oxigénio no sangue, na síntese de DNA e produção de energia $(14,15)$. O Fe é essencial para o normal desenvolvimento do cérebro e função cognitiva, crescimento, produção de neurotransmissores e mielinização (11). As consequências da deficiência de $\mathrm{Fe}$ incluem diminuição do desempenho físico e cognitivo (concentração e memória de curto prazo), depressão e fadiga $(10,11)$. As consequências sociais e económicas incluem aumento da mortalidade perinatal, baixa produtividade laboral e aumento das necessidades energéticas (10).

Nas leguminosas, o Fe é armazenado em complexos de ferritina, mas apenas uma parte deste está biodisponível. Assim, estão a ser investigadas estratégias que aumentem a sua biodisponibilidade e reduzam a concentração de anti-nutrientes (4).

\section{METODOLOGIA}

Esta revisão foi realizada nas bases de dados "ScienceDirect" e "Scholar Google" usando simultaneamente os seguintes descritores em inglês: "iron bioavailability", "legumes" e "phytate". A partir das fontes mencionadas, dos 1630 artigos publicados entre 2002 e 2020, foram excluídos todos os resumos e incluídos artigos de revisão para melhor compreensão dos conceitos abordados, porém dando preferêcia a artigos de pesquisa. Para além disto, foram excluídos estudos em animais e incluídos estudos em seres humanos e estudos com métodos laboratoriais, como por exemplo, a cromatografia líquida de alta pressão (HPLC) ou a espetrofotometria. Com base nestes critérios de exclusão e inclusão, selecionaram-se 53 artigos a partir da leitura do título e do resumo. Após a leitura e análise dos artigos selecionados e atendendo ao seu nível de pertinência para a temática da revisão, utilizaram-se 25 artigos para a elaboração desta.

\section{Anti-nutrientes e Fitato}

Os anti-nutrientes ou fatores anti-nutricionais são compostos sintetizados nos alimentos, pelo metabolismo normal das espécies. Estas substâncias, por si só ou por meio dos seus produtos metabólicos, interferem na utilização metabólica de alimentos, atuando para reduzir a digestão, absorção e utilização de nutrientes, e podem produzir outros efeitos adversos. Algumas destas substâncias conhecidas como "metabolitos secundários" são biologicamente ativas e provocam efeitos biológicos e fisiológicos adversos quando ingeridos pelo Homem e pelos animais, enquanto que outras são aplicadas na nutrição e como agentes farmacologicamente ativos. A concentração dos constituintes anti-nutricionais nas leguminosas variam consoante as espécies da planta e métodos de processamento pós-colheita $(16,17)$.

O fitato tem sido dominante em comparação com outros inibidores da biodisponibilidade de minerais, como as fibras dietéticas ou os taninos (18). Foi sugerido que doses baixas de fitatos reduzem o risco de cancro do cólon devido ao seu efeito antioxidante e pré-biótico e porque se ligam a enzimas como as amilases, para que parte do amido não digerido atinja o intestino $(19,20)$.

O fitato, complexo de ácido fítico (inositol fosfato) e mineral, é um agente quelante e é a principal forma de armazenamento de fósforo e inositol em leguminosas $(9,15,18)$. Dos diferentes tipos de inositol fosfato, como o mio-inositol hexafosfato e inositol pentafosfato, o inositol hexafosfato é o mais abundante nas plantas (15). A fitase é uma fosfatase específica de fitato que hidrolisa o fitato em inositol e ortofosfato livre e liberta minerais (18).

Sob condições ácidas no estômago, o ácido fítico, por possuir alta densidade de grupos fosfato carregados negativamente, liga-se aos iões minerais, formando complexos estáveis e insolúveis. No intestino delgado, estes complexos precipitam devido ao aumento do $\mathrm{pH}$ durante a passagem do estômago para o intestino, reduzindo a biodisponibilidade dos minerais, pois não são facilmente absorvidos pelo trato gastrointestinal $(6,16,20,21)$. Depois de chegar ao cólon, o fitato é degradado pela fitase produzida pela microbiota intestinal, sendo que esta degradação é afetada pelo cálcio e magnésio. Uma elevada ingestão de cálcio e magnésio afeta a solubilidade do fitato no trato gastrointestinal e reduz a acessibilidade do fitato para hidrólise enzimática (21).

O ácido fítico também inibe a ação da tirosinase gastrointestinal, tripsina, pepsina, lípase e amílase (16).

A previsão da biodisponibilidade de um mineral em alimentos com fitatos é difícil de fazer devido às interações entre minerais e ácido fítico, à dose de ácido fítico, atividade da fitase endógena, condições de processamento, digestibilidade do alimento e estado fisiológico e intestinal individual (15).

A razão molar fitato/mineral é uma ferramenta para prever a biodisponibilidade dos minerais (15). O fitato começa a perder o seu efeito inibitório sobre o Fe quando a razão molar é inferior a 1. É importante referir que diferentes alimentos são consumidos numa refeição e que esta razão molar pode ser facilmente alterada $(15,20)$, podendo haver um efeito inibitório forte até quando a razão fitato/ Fe é baixa como 0,2. O teor de fitato deve ser reduzido, idealmente $\leq 25 \mathrm{mg} / 100 \mathrm{~g}$, para minimizar perdas de micronutrientes (20).

A biodisponibilidade de minerais, como o Fe, pode ser melhorada aumentando fatores alimentares, como o ácido ascórbico (vitamina C), que aumentem a absorção mineral no intestino (14). Outras estratégias para melhorar a biodisponibilidade e reduzir fatores anti-nutricionais 
são a demolha (15), a germinação $(6,7,18)$, a fermentação $(18,19)$, o tratamento térmico $(6,7,15,19,22)$ e os acidulantes alimentares (18). Apesar da fermentação e germinação implicarem determinadas propriedades organoléticas indesejáveis $(9,18)$.

\section{Estratégias para a Redução de Anti-nutrientes}

Muitos estudos têm contribuído para avaliar quais as estratégias mais eficazes e eficientes na redução do efeito dos anti-nutrientes, em particular, estratégias que reduzam o teor de fitato e que melhorem a biodisponibilidade mineral.

Lestienne et al. (2005) mostraram que a demolha reduziu o teor de Fe e de fitatos (entre os 17 e 28\%) e não melhorou a razão molar fitato/Fe nem a biodisponibilidade deste mineral (9). Habiba (2002) verificou que a cozedura das envilhas reduziu o teor de ácido fítico em maior quantidade (47,9\%) na cozedura de 40 minutos (3). No estudo de Brigide et al. (2019), a demolha e a cozedura dos feijões reduziu a quantidade de inositol hexafosfato, sendo o tratamento dos feijões demolhados, cozidos e secos/desidratados o mais promissor para a biodisponibilidade do Fe (15). Assim, concluíram que, a demolha por si só não foi um bom método para melhorar a biodisponibilidade mineral, mas pode ser útil se combinada com outros tratamentos ou condições otimizadas de demolha (9).

Pal et al. (2017) e Luo et al. (2013) verificaram que a germinação reduziu o teor de ácido fítico em 40-59\% e melhorou a biodisponibilidade de Fe em 4 vezes mais nas favas e soja, respetivamente $(7,18)$. No estudo de Sant'Ana et al. (2019), verificaram que a razão fitato/Fe estava abaixo do valor considerado prejudicial para a biodisponibilidade de $\mathrm{Fe}$, demonstrando que a cozedura e germinação reduzem fitatos e melhoram a biodisponibilidade do $\mathrm{Fe}(6)$.

Lestienne et al. (2005) e Habiba (2002) verificaram que na demolha, cozedura sob pressão e na cozedura durante 40 min, as leguminosas mostraram baixo teor de proteínas, bem como baixos níveis de fitato. Isto mostra que a demolha e o cozimento melhoram a digestibilidade das leguminosas, devido à redução do conteúdo em ácido fítico e devido ao efeito do calor na estrutura tridimensional das proteínas. No entanto, o processamento também pode causar uma diminuição na digestibilidade das proteínas, por escurecimento não enzimático e formação de complexos entre proteínas e fitatos $(3,9)$.

A demolha, o cozimento e a germinação são processos simples, comuns e eficazes que reduzem o conteúdo de fitatos e melhoram a biodisponibilidade mineral das leguminosas $(7,9,18)$. No entanto, é importante referir que, os efeitos da demolha, cozimento e germinação nos componentes anti-nutricionais dependem do tipo de leguminosa e das condições usadas durante os processos, como o tempo, temperatura, teor de humidade, $\mathrm{pH}$ e presença ou ausência de luminosidade $(3,6,15)$.

Para além disso, existem outros fatores que afetam a biodisponibilidade, como potenciadores de absorção de Fe (aminoácidos, carne, aves e peixe) e inibidores da absorção de Fe (fitato, proteína da gema do ovo e fosvitina da gema do ovo, caseína, conglicinina, albumina e certos polifenóis) (15).

\section{Estratégias para Prevenir Deficiência de Ferro}

As estratégias nutricionais para prevenir a deficiência de Fe incluem diversidade alimentar, suplementação de Fe, fortificação alimentar e a biofortificação, uma alternativa sustentável, barata e eficaz $(10,23)$. A biofortificação visa aumentar os níveis de micronutrientes, através de práticas agronómicas, melhoria genética convencional de plantas ou biotecnologia moderna. Esta técnica pode ser benéfica para populações vulneráveis, mesmo que o aumento de micronutrientes seja pequeno $(11,15,23)$.
O aumento da concentração de Fe nas culturas alimentares pode não resultar num aumento proporcional de Fe absorvido, pois alimentos com alto teor de Fe também podem ter concentrações aumentadas ou diminuídas de inibidores ou intensificadores da absorção de Fe. Portanto, é necessário analisar a concentração e a biodisponibilidade de Fe, em culturas alimentares com altas concentrações de minerais $(15,23)$.

Junqueira-Franco et al. (2018) testaram a absorção do Fe de feijão, em voluntários, não anémicos. A concentração de Fe do feijão comum foi de $56,554 \mathrm{mg} / \mathrm{kg}$ e do feijão biofortificado foi de $72,966 \mathrm{mg} / \mathrm{kg}$, cerca de $29 \%$ mais que o feijão comum. O consumo de Fe dos feijões comuns foi de 5,65 mg e de 7,29 mg para os feijões fortificados, pelo que a absorção de Fe não diferiu estatisticamente entre os dois grupos. Provavelmente, a concentração de Fe no feijão biofortificado não foi suficiente para aumentar a biodisponibilidade (23).

Haas et al. (2016) avaliaram a eficácia de feijão biofortificado com Fe, numa população com deficiência de Fe. Enquanto que o feijão comum continha 50mg Fe/kg, o feijão biofortificado continha 86mg Fe/kg, ou seja, continha $71 \%$ mais Fe que os feijões comuns. A ingestão de Fe a partir do feijão foi de 14,5 mg/dia no feijão biofortificado e de $8,6 \mathrm{mg} /$ dia no feijão comum e o Fe absorvido foi de 1,06 mg/dia e de $0,79 \mathrm{mg} / \mathrm{dia}$, respetivamente. Isto representa $75 \%$ e $56 \%$ da dose diária recomendada. Os feijões biofortificados com Fe aumentaram significativamente a hemoglobina, as concentrações de ferritina sérica e o Fe corporal total. Os resultados deste estudo também sugerem que o consumo de feijão biofortificado melhora as consequências da deficiência de Fe, como capacidade física e função cognitiva (10).

\section{ANÁLISE CRÍTICA}

O ácido fítico é um dos inibidores dominantes da biodisponibilidade de minerais e atua, ligando-se a minerais como o Fe, dificultando a sua absorção $(6,16,18,20,21)$. Assim, pode haver alteração do status de Fe e dos processos metabólicos nos quais o Fe intervém, como é o caso da síntese de hemoglobina, hormonas, DNA, tecido conjuntivo e muscular e transporte de oxigénio no sangue $(4,14)$. Consequentemente, o desempenho físico e cognitivo, o crescimento, o desenvolvimento cerebral, a produção de neurotransmissores e a mielinização podem ser afetados $(10,11,14,15)$.

A redução dos fitatos nas leguminosas durante a demolha, germinação e cozedura é atribuída à síntese e ativação de fitases endógenas e à libertação dos iões fitato que se difundem na água da demolha e cozedura $(7,15,18,19)$. Isto melhora a qualidade nutricional dos alimentos e aumenta o seu conteúdo nutritivo e digestibilidade, podendo duplicar a biodisponibilidade de Fe (18).

Lestienne et al. (2015) verificaram que níveis baixos de fitatos estavam associados a baixos teores de proteína. Isto pode ser explicado pelo facto do ácido fítico formar um complexo com aminoácidos, reduzindo a sua digestibilidade e biodisponibilidade. Como a demolha, germinação e tratamento térmico reduzem o conteúdo de fitato, vão também reduzir os complexos ácido fítico-aminoácido, aumentando assim os aminoácidos disponíveis e a digestibilidade das proteínas (3, 5, 9, 15, 16).

A biodisponibilidade do Fe nas leguminosas pode ser aumentada através da melhoria convencional de plantas, sem sacrificar as características agronómicas (24). A biofortificação em Fe, para além de aumentar o status de $\mathrm{Fe}$, pode prevenir as consequências da deficiência de Fe relacionadas com a função cognitiva, memória e atenção, capacidade física e resistência $(10,11)$.

Para planear programas e intervenções de saúde pública, devem comparar-se os efeitos da biofortificação, da fortificação e da suplementação. Por exemplo, num ambiente com elevada deficiência de Fe, a suplementação é a abordagem a curto prazo, enquanto que a 
fortificação e biofortificação são estratégias económicas e sustentáveis de manutenção a longo prazo $(11,25)$.

Estudos futuros serão necessários para populações de risco e para avaliar os resultados funcionais, como a performance física e cognitiva. Para, deste modo, desenvolver técnicas como a biofortificação, para diminuir as deficiências de Fe e melhorar a saúde das populações (11, 24). Além disso, são essenciais estudos da biofortificação de outras variedades de leguminosas, para além do feijão já estudado, e da combinação de vários alimentos biofortificados com Fe, cereais (trigo, arroz, milho) e leguminosas (feijão, grão, lentilha, envilha) (24).

\section{CONCLUSÕES}

A pesquisa científica e a análise dos artigos selecionados permitiram entender como se processa a metabolização do fitato, quais os seus efeitos e quando a razão fitato/Fe é maior que 1, o fitato inibe a absorção de Fe. Neste artigo de revisão, foram também tidas em consideração, as estratégias para melhorar a biodisponibilidade de minerais como o Fe. Foi possível entender que os processos de demolha, germinação e tratamento térmico reduzem o conteúdo de fitato e aumentam a atividade da fitase, melhorando a biodisponibilidade mineral das leguminosas. Enquanto que, a biofortificação aumenta os níveis de micronutrientes, beneficiando a saúde de populações vulneráveis, a longo prazo.

\section{REFERÊNCIAS BIBLIOGRÁFICAS}

1. Thompson HJ. Dietary Bean Consumption and Human Health. Nutrients [Internet]. 2019 Dec 17;11(12):3074. Available from: https://www.mdpi.com/2072-6643/11/12/3074. 2. Kumar S, Pandey G. Biofortification of pulses and legumes to enhance nutrition. Heliyon [Internet]. 2020 Mar;6(3):4-9. Available from: https://linkinghub.elsevier.com/ retrieve/pii/S2405844020305272.

3. Habiba R. Changes in anti-nutrients, protein solubility, digestibility, and $\mathrm{HCl}$ extractability of ash and phosphorus in vegetable peas as affected by cooking methods. Food Chem [Internet]. 2002 May 1 [cited 2020 Feb 27];77(2):187-92. Available from: https://linkinghub.elsevier.com/retrieve/pii/S0308814601003351.

4. Castro-Guerrero NA, Isidra-Arellano MC, Mendoza-Cozatl DG, Valdés-López O. Common Bean: A Legume Model on the Rise for Unraveling Responses and Adaptations to Iron, Zinc, and Phosphate Deficiencies. Front Plant Sci [Internet]. 2016 May 3;7(May):391-417. Available from: https://www.frontiersin.org/articles/10.3389/ fpls.2016.00600/full.

5. Oliveira AP, Naozuka J. Chemical Speciation of Iron in Different Varieties of Beans (Phaseolus vulgaris L.): Cooking Effects. J Braz Chem Soc [Internet]. 2015;26(10):21449. Available from: http://www.gnresearch.org/doi/10.5935/0103-5053.20150201.

6. Sant' Ana CT, Antunes PT, Reis TC, Váz-Tostes MG, Meira EF, Costa NMB. Bioaccessibility and bioavailability of iron in biofortified germinated cowpea. J Sci Food Agric [Internet]. 2019 Nov 6;99(14):6287-95. Available from: https://onlinelibrary.wiley. com/doi/abs/10.1002/jsfa.9902.

7. Pal RS, Bhartiya A, Yadav P, Kant L, Mishra KK, Aditya JP, et al. Effect of dehulling, germination and cooking on nutrients, anti-nutrients, fatty acid composition and antioxidant properties in lentil (Lens culinaris). J Food Sci Technol [Internet]. 2017 Mar 7;54(4):909-20. Available from: http://link.springer.com/10.1007/s13197-016-2351-4. 8. Jager I, Borgonjen-van den Berg KJ, Giller KE, Brouwer ID. Current and potential role of grain legumes on protein and micronutrient adequacy of the diet of rural Ghanaian infants and young children: using linear programming. Nutr J [Internet]. 2019 Dec 21 [cited 2020 Feb 27];18(1):12. Available from: http://www.ncbi.nlm.nih.gov/pubmed/30791898. 9. Lestienne I, Icard-Vernière C, Mouquet C, Picq C, Trèche S. Effects of soaking whole cereal and legume seeds on iron, zinc and phytate contents. Food Chem [Internet]. 2005 Feb 1 [cited 2020 Feb 27];89(3):421-5. Available from: https://linkinghub.elsevier. com/retrieve/pii/S0308814604002298.

10. Haas JD, Luna S V, Lung'aho MG, Wenger MJ, Murray-Kolb LE, Beebe S, et al. Consuming Iron Biofortified Beans Increases Iron Status in Rwandan Women after 128
Days in a Randomized Controlled Feeding Trial. J Nutr [Internet]. 2016 Aug 1;146(8):158692. Available from: https://academic.oup.com/jn/article/146/8/1586/4584652.

11. Finkelstein JL, Fothergill A, Hackl LS, Haas JD, Mehta S. Iron biofortification interventions to improve iron status and functional outcomes. Proc Nutr Soc [Internet]. 2019 May 30;78(02):197-207. Available from: https://www.cambridge.org/core/ product/identifier/S0029665118002847/type/journal_article.

12. Weinborn V, Pizarro F, Olivares M, Brito A, Arredondo M, Flores S, et al. The Effect of Plant Proteins Derived from Cereals and Legumes on Heme Iron Absorption. Nutrients [Internet]. 2015 Oct 30;7(11):8977-86. Available from: http://www.mdpi. com/2072-6643/7/11/5446.

13. Anitha S, Govindaraj M, Kane-Potaka J. Balanced amino acid and higher micronutrients in millets complements legumes for improved human dietary nutrition. Cereal Chem [Internet]. 2020 Jan 21;97(1):74-84. Available from: https://onlinelibrary. wiley.com/doi/abs/10.1002/cche.10227.

14. Singh P, Prasad S, Aalbersberg W. Bioavailability of Fe and Zn in selected legumes, cereals, meat and milk products consumed in Fiji. Food Chem [Internet]. 2016 Sep 15 [cited 2020 Feb 27];207:125-31. Available from: https://linkinghub.elsevier.com/ retrieve/pii/S0308814616303806.

15. Brigide P, Toledo NMV, López-Nicolás R, Ros G, Saseta CF, Carvalho RV. Fe and Zn in vitro bioavailability in relation to antinutritional factors in biofortified beans subjected to different processes. Food Funct [Internet]. 2019;10(8):4802-10. Available from: http:// xlink.rsc.org/?DOI=C9FO00199A.

16. Tadele Y. Important Anti-Nutritional Substances and Inherent Toxicants od Feeds. Food Sci Qual Manag [Internet]. 2015;36:40-7. Available from: https://www.iiste.org/ Journals/index.php/FSQM/article/view/19778.

17. Olanipekun OT, Omenna EC, Olapade OA, Suleiman P, Omodora OG. Effect of Boiling and Roasting on the Nutrient Compostion of Kidney Beans Seed Flour. Sky J Food Sci [Internet]. 2015;4(2):024-9. Available from: http://www.skyjournals.org/sjfs/ pdf/2015/Mar/Olanipekun et al pdf..pdf.

18. Luo Y-W, Xie W-H, Jin X-X, Wang Q, He Y-J. Effects of germination on iron, zinc, calcium, manganese, and copper availability from cereals and legumes. CyTA - J Food [Internet]. 2013 Jan 2;12(1):22-6. Available from: http://www.tandfonline.com/doi/abs /10.1080/19476337.2013.782071.

19. Margier M, Georgé S, Hafnaoui N, Remond D, Nowicki M, Chaffaut L, et al. Nutritional Composition and Bioactive Content of Legumes: Characterization of Pulses Frequently Consumed in France and Effect of the Cooking Method. Nutrients [Internet]. 2018 Nov 4;10(11):1668. Available from: http://www.mdpi.com/2072-6643/10/11/1668.

20. Udomkun P, Tirawattanawanich C, llukor J, Sridonpai P, Njukwe E, Nimbona P, et al. Promoting the use of locally produced crops in making cereal-legume-based composite flours: An assessment of nutrient, antinutrient, mineral molar ratios, and aflatoxin content. Food Chem [Internet]. 2019 Feb 20;286:651-8. Available from: https://linkinghub.elsevier.com/retrieve/pii/S0308814619303693.

21. Lo D, Wang H, Wu W-J, Yang R-Y. Anti-nutrient components and their concentrations in edible parts in vegetable families. CAB Rev Perspect Agric Vet Sci Nutr Nat Resour [Internet]. 2018 Aug 1;13(015). Available from: http://www.cabi.org/cabreviews/ review/20183230385.

22. Omari R. Nutrition knowledge and food consumption practices and barriers in rural Ghana: The case of foods for preventing vitamin A and iron deficiencies. African J Food, Agric Nutr Dev [Internet]. 2017 Mar 22;17(01):11639-56. Available from: http://ajfand. net/Nolume17/No1/Omari15815.pdf.

23. Junqueira-Franco MVM, Dutra de Oliveira JE, Nutti MR, Pereira HS, Carvalho JLV, Abrams SA, et al. Iron absorption from beans with different contents of iron, evaluated by stable isotopes. Clin Nutr ESPEN [Internet]. 2018 Jun;25:121-5. Available from: https://linkinghub.elsevier.com/retrieve/pii/S2405457717304941.

24. Boy E, Haas J, Petry N, Cercamondi Cl, Gahutu J, Mehta S, et al. Efficacy of IronBiofortified Crops. African J Food, Agric Nutr Dev [Internet]. 2017;17(2):11879-92. Available from: https://www.ajfand.net/Volume17/No2/Biofortification Issue Chapter 3.pdf. 25. Bouis HE, Hotz C, McClafferty B, Meenakshi J V., Pfeiffer WH. Biofortification: A New Tool to Reduce Micronutrient Malnutrition. Food Nutr Bull [Internet]. 2011 Mar 14;32(1):S3140. Available from: http://journals.sagepub.com/doi/10.1177/15648265110321S105. 